\title{
Coetaneous Presentation of Oral Leukoplakia and Oral Submucous Fibrosis with High Grade Dysplasia and Its Management with Lasers: A Case Report
}

\author{
Preethi $\mathbf{R}^{1}$, Malarkodi $\mathbf{T}^{2}$, Arvind Krishnamurthy ${ }^{3}$, Aravind Warrier $\mathrm{S}^{3}$
}

${ }^{1}$ Post Graduate, Department of Oral Medicine and Radiology, Sri Ramachandra Faculty of Dental Sciences, Sri

Ramachandra Institute of Higher Education and Research (DU), Chennai-600116, Tamilnadu, India

${ }^{2}$ Associate Professor, Department of Oral Medicine and Radiology, Sri Ramachandra Faculty of Dental Sciences, Sri

Ramachandra Institute of Higher Education and Research (DU), Chennai-600116, Tamilnadu, India

${ }^{3}$ Professor \& Head, Department of Surgical Oncology, Cancer Institute, Adyar, Chennai -600020, Tamilnadu, India

${ }^{4}$ Professor \& Head, Department of Oral Medicine and Radiology, Sri Ramachandra Faculty of Dental Sciences, Sri

Ramachandra Institute of Higher Education and Research (DU), Chennai-600116, Tamilnadu, India

*Corresponding Author: Dr. Malarkodi T.; tmalarkodi@sriramachandra.edu.in

Received: 10 July 2021;

Accepted: 31 July 2021;

Published: 02 August 2021

\begin{abstract}
Oral potentially malignant disorders (OPMD) are diverse lesions associated with an increased likelihood for malignant transformation to oral cancer. The most frequently encountered OPMD in clinical practice are Oral Leukoplakia and oral submucous fibrosis (OSMF). The use of tobacco is consistently linked with leukoplakia and areca/betel nut with OSMF. Histopathological features of oral epithelial dysplasia, its varying grades are of immense clinical significance to evaluate its ability for malignant transformation. We report a case of homogenous leukoplakia and OSMF, two oral potentially malignant disorders occurring at the same site with high-grade dysplasia and its management using lasers.
\end{abstract}

Keywords: Oral potentially malignant disorders, OPMD, Oral submucous fibrosis, Oral Leukoplakia, Oral epithelial dysplasia

\section{Introduction}

Oral Potentially Malignant Disorders (OPMDs) is the terminology used for an oral lesion or a condition that may have the potential risk to become malignant ${ }^{[1]}$. According to the theory of field cancerization, malignancy can arise anywhere in a clinically normal mucosa even when the OPMD is limited to a specific site ${ }^{[2]}$. The prevalence of OPMD worldwide is $4.47 \%$, with a male predilection ${ }^{[3]}$.

The WHO definition explains oral leukoplakia as a white lesion that cannot be clinically distinguished and delineated as any other oral lesion. Tobacco usage in the form of smoking is the main etiology. This disorder is of two types, homogeneous and nonhomogeneous ${ }^{[4]}$. The prevalence of leukoplakia is $2.6 \%$ and the overall malignant transformation rate is $1.5 \%$ to $34 \%$. The Non-homogeneous variety has shown a higher malignant transformation rate of $14.5 \%$ compared to the homogenous variety which shows $3 \%{ }^{[1]}$. Although oral leukoplakia can occur at any site, buccal mucosa is considered to be the most common, followed by the gingiva, tongue, and floor of the mouth. Common sites for malignant transformation are the tongue $(24.22 \%)$, followed by the floor of the mouth $(14.85 \%)^{[1]}$.

OSMF was initially termed atrophica "idiopathica (Tropica) mucosae oris" ${ }^{[5]}$. It affects the oral mucosa, pharynx, and upper two-thirds of the esophagus leading to progressive trismus and dysphagia due to esophageal involvement. Arecoline present in the areca nut is considered the major etiological factor in OSMF. The malignant transformation and prevalence rate of OSMF is $7-13 \%$ and $2.01 \%{ }^{[4,5]}$.

WHO (2005) uses the term epithelial precursor lesions ${ }^{[6]}$ and defines it as "altered epithelium with an increased likelihood for progression to squamous cell carcinoma", where epithelial dysplasia is signified as "altered" ${ }^{[7]}$. Mehanna et al reported $10.3 \%$ as a malignant transformation rate for mild/moderate dysplasias and $24.1 \%$ for the severe variety of oral epithelial dysplasia (OED) ${ }^{[8]}$. The malignant progression rates for non-dysplastic lesions were $0.012 \%$, and $6 \%, 18 \%$, and $39 \%$ for lesions with mild, moderate, and severe dysplasia, respectively. The severity of OED is a significant prognostic indicator that supports the fact that OED 
grading could be a vital marker to assess the risk of malignant progression ${ }^{[1]}$.

\section{Case report}

A 53-year male patient came with a chief complaint of mobile right lower back teeth for the past 1 month. The patient is a known case of type-II Diabetes Mellitus, hypertensive for the past 5 years, and under oral hypoglycemic agents and candesartan (angiotensin II receptor blocker). The patient has a history of smoking 20 beedi per day for 10 years.; chewing tobacco with betel nut and at times with Gutka 15 times a day for 10 years and has the habit of pouching the quid in the right cheek and spits it out after 30 minutes; history of alcohol consumption once a day for 10 years.

A single raised, well-demarcated, white plaque of size $4 * 3 \mathrm{~cm}$ in the right buccal mucosa was evident on clinical examination. It was extending anteriorly $1 \mathrm{~cm}$ short of right commissure, posteriorly till the retromolar region, superiorly 0.5 $\mathrm{cm}$ below the upper buccal sulcus, and inferiorly involving the lower buccal sulcus, alveolar mucosa, and marginal gingiva in relation to 46,47 . The lesion was slightly raised, leathery and tender over the lower buccal sulcus in relation to 47 . Vertical fibrous bands were palpable in the right buccal mucosa. (Fig. 1) Palatal mucosa was blanched with loss of gag on palpation. Left buccal mucosa, uvula, labial mucosa, tongue, mouth opening, and tongue movements were within normal limits. A single right submandibular lymph node was palpable, measuring less than $1 \mathrm{~cm}$ in diameter, freely mobile, firm, and nontender. A provisional diagnosis of Homogenous leukoplakia and OSMF involving the right buccal mucosa was given.

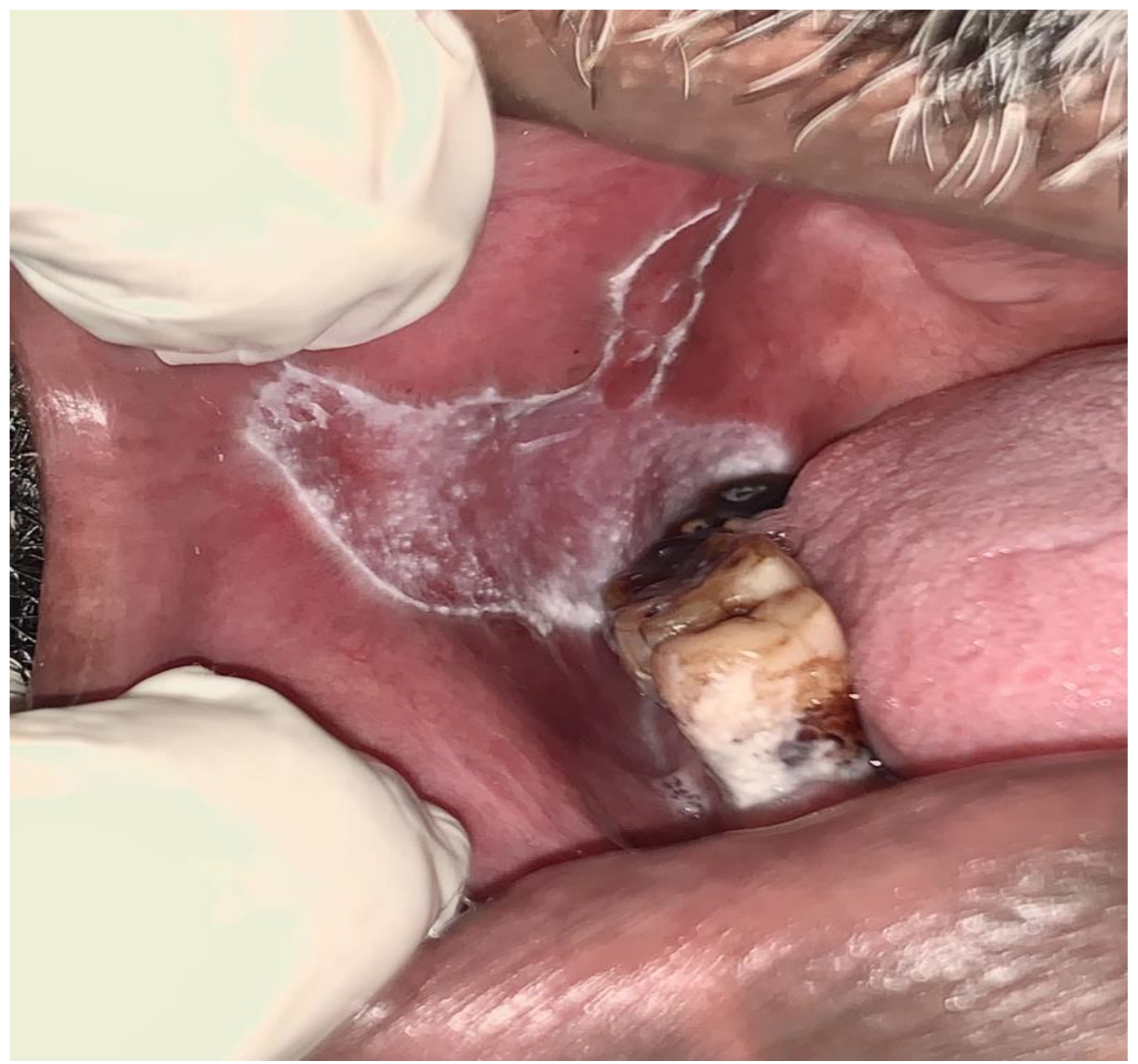

Figure 1: Right buccal mucosa, showing homogenous leukoplakia with underlying submucous fibrosis.

Hemoglobin level was $12 \mathrm{gms} / \mathrm{dl}$, fasting and postprandial blood glucose levels were $96 \mathrm{mg} / \mathrm{dl}$ and $158 \mathrm{mg} / \mathrm{dl}$. Incisional biopsy was done where the histopathological features showed an ortho keratinized epithelium with few areas of atrophy and juxtaepithelial hyalinization, collagen of varying density with hyperplasia, and hypertrophy of cells without invasion of the basement membrane suggesting Oral submucous fibrosis with high-grade dysplasia and other features correlating with a clinical diagnosis of Homogenous leukoplakia. (Fig. 2) Ultrasound evaluation of the neck revealed prominent level IB and II nodes with fatty hilum preserved. (Fig. 3) Considering the clinical, histopathological, and ultrasound features, the final diagnosis was Oral submucous fibrosis with high-grade dysplasia and Homogenous leukoplakia.

Potassium Titanyl Phosphate (KTP) laser excision of the lesion was done and the patient is under regular follow-up. (Fig. 4) 


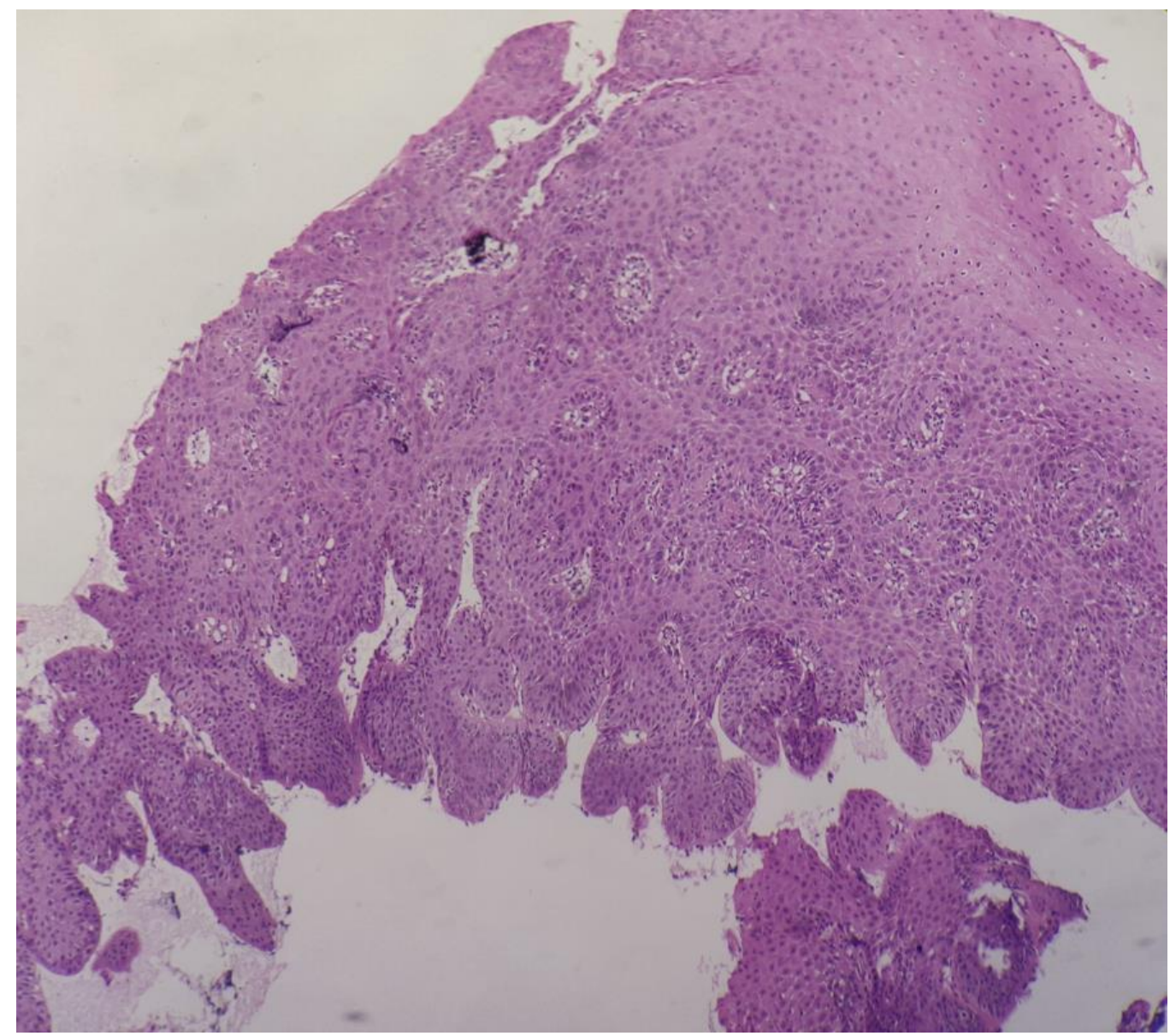

Figure 2: H\&E stain showing features of OSMF with high-grade epithelial dysplasia
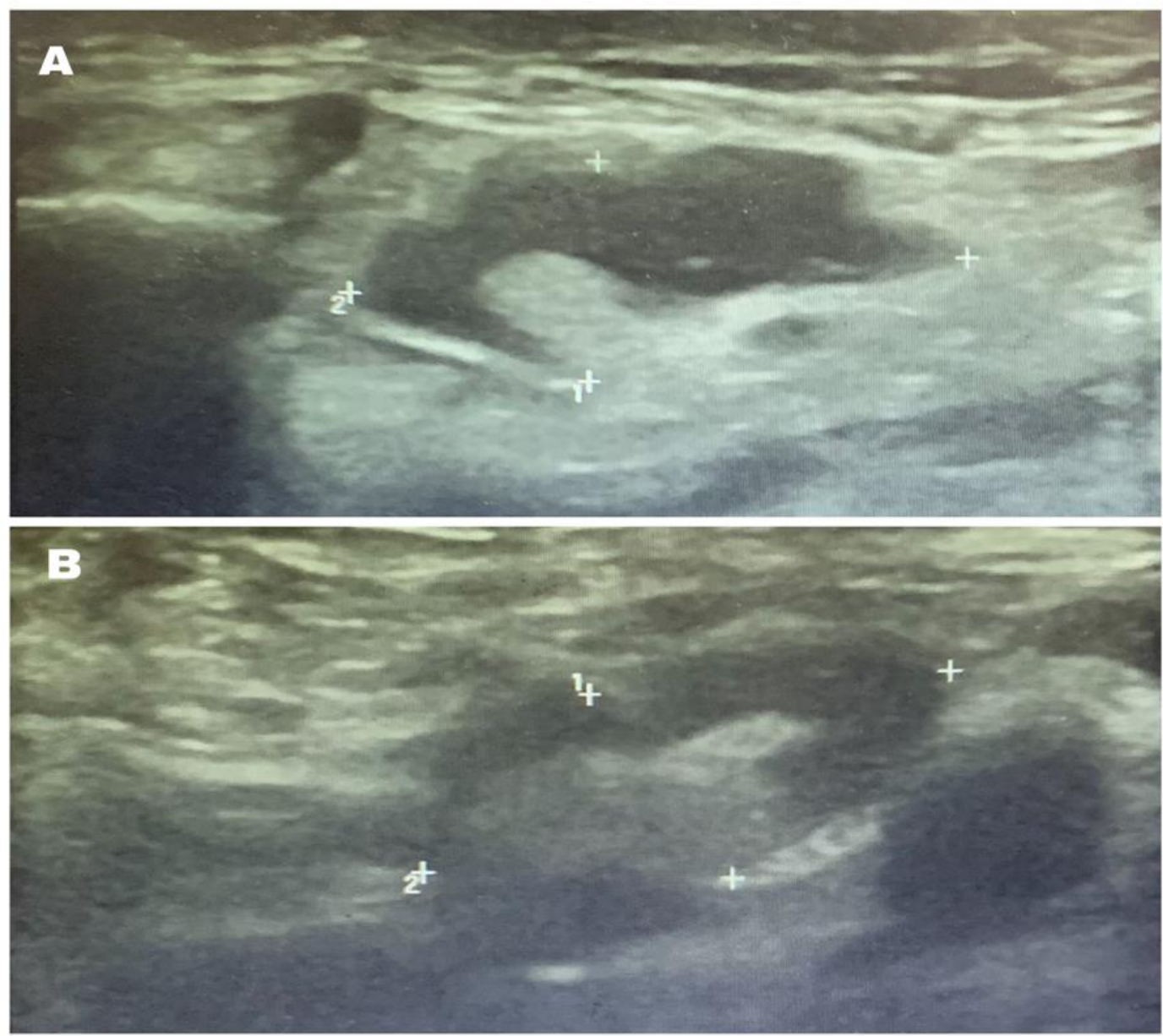

Figure 3: 2D mode high-resolution ultrasound of neck showing (A) prominent level IB and (B) level II cervical lymph nodes with the preserved fatty hilum 


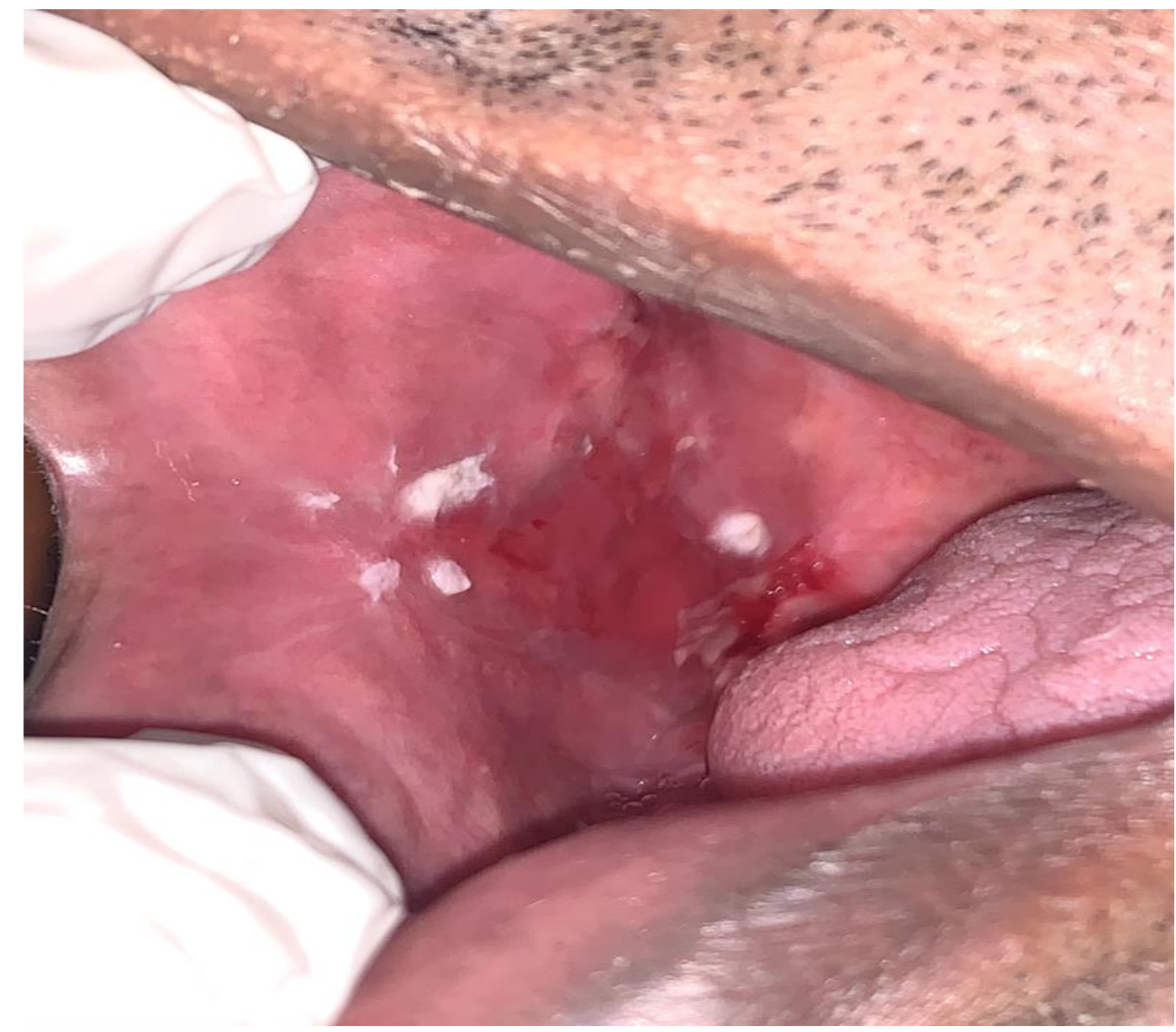

Figure 4 - Post-operative view of the lesion after excision with Potassium Titanyl Phosphate (KTP) laser.

\section{Discussion}

OPMDs are statistically more likely to become malignant ${ }^{[1,2]}$. Parameters and clinical features related to increased risk of malignant progression of OPMD are lesion size over $200 \mathrm{~mm}^{2}$, nonhomogeneous texture, red or speckled in color, and when the location is either tongue or floor of the mouth. Although these clinical criteria may help in the assessment of risk, biopsy followed by histopathologic examination is considered to be the gold standard for the diagnosis ${ }^{[1]}$.

Homogeneous leukoplakia appears as a white plaque-like lesion that can be flat, raised, or wrinkled with surface cracks or fissures. Non-homogeneous variety has varied presentations which includes speckled or nodular or erythroleukoplakia, verrucous leukoplakia and verrucous proliferative variety. Microscopically, $50 \%$ of these lesions may be ortho or parakeratotic with acanthosis, varying degrees of chronic inflammatory infiltrates in lamina propria, and various degrees of epithelial dysplasia, rest shows non-specific hyperplasia and hyperkeratosis ${ }^{[1,4]}$.

The characteristic features of OSMF are altered texture, blanching, atrophic tongue, restricted tongue movements, trismus, sunken cheeks, vertical fibrous bands, shrunken uvula, burning sensation, ulceration, and pain. In the more advanced stages, fibrosis makes the cheek, tongue, thick and rigid causing difficulty in mastication, mouth opening, tongue movement, speech, swallowing, and maintaining oral hygiene ${ }^{[5]}$. Microscopic features of OSMF are, juxta-epithelial hyalinization, bands of collagen fibers, orienting horizontally or vertically with inflammatory infiltrate primarily with neutrophils, eosinophils, decreased vascularization, atrophic changes, and dysplastic features ${ }^{[5]}$.

The relationship between epithelial dysplasia and the risk of malignant transformation makes dysplasia grading necessary as the risk of malignancy is in a pari passu with the severity of dysplasia [9]. The WHO (2017) diagnostic features and grading of oral epithelial dysplasia is based on the architectural and cytological changes ${ }^{[10]}$. The architectural changes include "irregular epithelial stratification, loss of polarity of basal cells, drop-shaped rete ridges, increased number of mitotic figures, abnormal superficial mitosis, dyskeratosis (Premature keratinization in single cells), keratin pearls in rete ridges, and loss of epithelial cell cohesion". The cellular changes related to OED are "anisonucleosis (Abnormal variation in nuclear size), nuclear pleomorphism (Abnormal variation in nuclear shape), anisocytosis (Abnormal variation in cell size), cellular pleomorphism (Abnormal variation in cell shape), Increased nuclear-cytoplasmic ratio, atypical mitotic figures, increased number and size of nucleoli, and hyperchromasia". WHO (2017) now recommends a more objective three-tier grading of OED supporting the above features as mild, moderate, and severe dysplasia ${ }^{[10]}$.

Overall, only $50 \%$ of biopsy tissues show epithelial dysplasia of which $50 \%$ of severely $30 \%$, and $5 \%$ of moderate and mild dysplasia's advance to malignancy. The malignant transformation potential of all OPMD depends on the presence and grade of dysplasia. The overall malignant transformation rate of OED is 6-36\%, which indicates that not all dysplastic lesions progress to malignancy. Contemporary treatments include excision with a cold knife, carbon dioxide $\left(\mathrm{CO}_{2}\right)$ laser ablation, antioxidant therapy, immunomodulating therapy, or a combination of any of the above ${ }^{[11]}$.

Treatment methods for the homogeneous variety of oral leukoplakia, which isn't related to dysplasia, include cessation of the habit with regular follow-up, due to its low malignant transformation rate. Surgical excision cold knife, $\mathrm{CO}_{2} /$ neodymium-doped yttrium aluminum garnet (Nd: YAG)/ 
potassium-titanyl-phosphate (KTP) lasers, or a combination is normally reserved for those lesions with moderate to severe dysplasia and non-homogeneous leukoplakia, especially for the erythroleukoplakia and verrucous proliferative type ${ }^{[11]}$.

Several modes of therapy were planned and put forth in the past to scale back the signs and symptoms of OSMF. Cessation of the areca/betel nut chewing habit usually resolves the symptoms if diagnosed early, even before fibrosis may well be established. Supporting the severity grade, the management protocol for OSMF has been categorized as conservative and surgical. Conservative management for mild and moderate groups includes medical therapy to modulate inflammation, and immunity, to promote blood flow, to induce fibrinolysis, antioxidants, nutrients, micronutrients, vitamins, mineral supplements, and physical therapy. Surgical intervention is suggested for patients with severe trismus or with dysplastic changes in histopathology ${ }^{[12]}$.

Alternatively, surgical fibrotomy can be performed using lasers as they are less invasive, cause less bleeding, scarring with quick healing and short operating time ${ }^{[13]}$. The most common laser used for band excision is $\mathrm{CO}_{2}$ lasers. For primary incision, Erbium YAG (Er-YAG) laser, KTP 532, and the diode lasers are used ${ }^{[14]}$.

The angiolytic laser ${ }^{[15]}$ KTP-532 features a wavelength similar to visible light $(532 \mathrm{~nm})$, delivered via optical fibers, doesn't require an aiming beam, and is selectively absorbed by hemoglobin ${ }^{[16]}$. Its use in OPMD lies in the incontrovertible fact that it reduces postoperative edema and pain ${ }^{[15]}$. The advantage of the KTP-532 laser produces a zone of coagulation on either side of the incision and seals the edges of the wounds, making it a fast, well-tolerated, minimally invasive method. With this approach, external incisions and scars are avoided. The main disadvantage of the KTP-532 technique is the cost factor and causes more charring in comparison with water-absorbed lasers and superficial char may have to be manually removed to treat the deeper segment of the lesion ${ }^{[16]}$.

A study performed by Thomson et al. on 590 patients where excision of OPMD was done with $\mathrm{CO}_{2}$ laser exhibited dysplasia and carcinoma in situ in $88.5 \%$, and malignant transformation in $16.8 \%$, after a mean follow-up period of 7.3 years [17]

Mogedas- Vegara et al. in a retrospective study of 65 oral leukoplakia patients treated with $\mathrm{CO}_{2}$ laser, showed a recurrence rate of $33.8 \%$ and $15.4 \%$ malignant transformation ${ }^{[18]}$.

Presently, there are no widely recognized, specific guidelines to suggest the frequency and follow-up for OED patients, taking into consideration factors like field cancerization theory, age, gender, areca nut/tobacco use, site and size of the lesion, degree of dysplasia, uncertainty associated with malignant transformation and recurrence rate follow-up should be made more regular and frequent for such lesions ${ }^{[11]}$.

\section{Conclusion}

Oral potentially malignant disorders associated with epithelial dysplasia have variable rates of malignant transformation, hence interception of preventable risk factors like tobacco/areca/betel nut should be prioritized at all stages. We present a case of homogenous leukoplakia and oral submucous fibrosis with highgrade dysplasia, an occurrence of two potentially malignant disorders at the same site making the patient twice more susceptible to malignant transformation. After successful treatment with KTP-532 laser, and one-year follow-up to date, the patient didn't exhibit any signs of recurrence or malignant transformation.
Early diagnosis and swift management of OPMDs may prevent the lesion from advancing to malignancy.

\section{Consent to Participate}

Written consent has been obtained from the patient to publish the clinical details and pictures of the oral cavity.

\section{List of Abbreviations}

OPMD: Oral potentially malignant disorders

OSMF: Oral submucous fibrosis

OED: Oral epithelial dysplasia

KTP: Potassium Titanyl Phosphate

$\mathrm{CO}_{2}$ : Carbon dioxide

Nd: YAG: Neodymium-doped yttrium aluminum garnet

Er-YAG: Erbium YAG

\section{Conflict of Interest}

None

\section{Funding}

None

\section{Authors Contribution:}

P.R, M.T, S.A.W: clinically analyzed, diagnosed, and interpreted the patient data regarding Oral leukoplakia and OSMF.

A.K: Surgical management of the lesion

P.R: major contribution in drafting the article

M.T: critical revision of the article

All authors read and approved the final manuscript

\section{References}

[1] Speight PM, Khurram SA, Kujan O. "Oral potentially malignant disorders: risk of progression to malignancy". Oral surgery, oral medicine, oral pathology, and oral radiology. 2018 Jun 1;125(6):612-27.

[2] Van der Waal I. "Potentially malignant disorders of the oral and oropharyngeal mucosa; terminology, classification and present concepts of management". Oral oncology. 2009 Apr 1;45(4-5):317-23.

[3] Alsuwaiket Aa, Kazmi F, Mumtaz M. "Preventable Risk Factors Associated with Oral Potentially Malignant Disorders: A Study in Saudi Arabia". Asian Journal of Advances in Medical Science. 2020:31-9.

[4] Burket LW, Greenberg M, Click M. Burket's oral medicine 11th ed. BC Decker Inc. 2008:214-5.

[5] Ali FM, Patil A, Patil K, Prasant MC. "Oral submucous fibrosis and its dermatological relation". Indian dermatology online journal. $2014 \mathrm{Jul} ; 5(3): 260$.

[6] Sarode SC, Sarode GS, Tupkari JV. "Oral potentially malignant disorders: A proposal for terminology and definition with review of literature". Journal of oral and maxillofacial pathology: JOMFP. 2014 Sep;18(Suppl 1):S77.

[7] Barnes L, Eveson JW, Reichart P, Sidransky D, editors. Pathology and Genetics of Head and Neck Tumours. Lyon: IARC Press; 2005. World Health Organization Classification of Tumours; pp. 283-328. 
[8] Mehanna HM, Rattay T, Smith J, McConkey CC. "Treatment and follow-up of oral dysplasia-a systematic review and meta-analysis". Head \& Neck: Journal for the Sciences and Specialties of the Head and Neck. 2009 Dec;31(12):1600-9.

[9] Fleskens S, Slootweg P. "Grading systems in head and neck dysplasia: their prognostic value, weaknesses, and utility". Head \& neck oncology. 2009 Dec;1(1):1-8.

[10] El-Naggar AK, Chan JK, Grandis JR, Takata T, Slootweg PJ. "WHO Classification of Head and Neck Tumors". WHO/IARC Classification of Tumours. 4th ed., Vol. 9. International Agency for Research on Cancer (IARC) press; 2017.

[11] Awadallah M, Idle M, Patel K, Kademani D. "Management update of potentially premalignant oral epithelial lesions". Oral surgery, oral medicine, oral pathology, and oral radiology. 2018 Jun 1;125(6):628-36.

[12] Arakeri, G., Rai, K. K., Boraks, G., Patil, S. G., Aljabab, A. S., Merkx, M. A. W., Carrozzo, M., \& Brennan, P. A. "Current Protocols in the Management of Oral Submucous Fibrosis: An Update". J Oral Pathol Med. 2017 Apr46(6), 418-423.

[13] Chaudhry Z, Gupta SR, Oberoi SS. "The efficacy of ErCr: YSGG laser fibrotomy in management of moderate oral submucous fibrosis: a preliminary study". Journal of maxillofacial and oral surgery. 2014 Sep 1;13(3):286-94.

[14] Sadat SA, Benzadid MS, Nusrath M, Rita SN. "Management of Oral Sub-Mucous Fibrosis: An update". Journal of Bangladesh College of Physicians and Surgeons. 2020 May 12;38(3):135-44.

[15] Novakovic D, Rickert S, Blitzer A. "Office-based laser treatment of oral premalignant lesions". Operative Techniques in Otolaryngology-Head and Neck Surgery. 2011 Jun 1;22(2):159-64.

[16] Hazarika P, Pillai S, Jacob SM, Punnoose SE, Roy A. "Application of potassium-titanyl-phosphate (KTP) laser in the excision of pyriform fossa hemangioma". American journal of otolaryngology. 2006 Mar 1;27(2):136-8.

[17] Thomson PJ, Goodson ML, Cocks K, Turner JE. "Interventional laser surgery for oral potentially malignant disorders: a longitudinal patient cohort study". International journal of oral and maxillofacial surgery. 2017 Mar 1;46(3):337-42.

[18] Mogedas-Vegara A, Hueto-Madrid JA, ChimenosKüstner E, Bescós-Atín C. "The treatment of oral leukoplakia with the $\mathrm{CO}_{2}$ laser: A retrospective study of 65 patients". Journal of Cranio-Maxillofacial Surgery. 2015 Jun 1;43(5):677-81. 